\title{
Normalized Mass Maps in Three-Dimensional Space Combining Kendrick-like Values With Chromatographic Separation for Enhanced Data Deconvolution.
}

Robert J. Strife $\dagger^{*}$ and Scott Campbell $\uparrow$, Jason Price $\uparrow$ and Safa Motlagh $\uparrow$

SUPPORTING INFORMATION FILE - PNG AND CAPTION

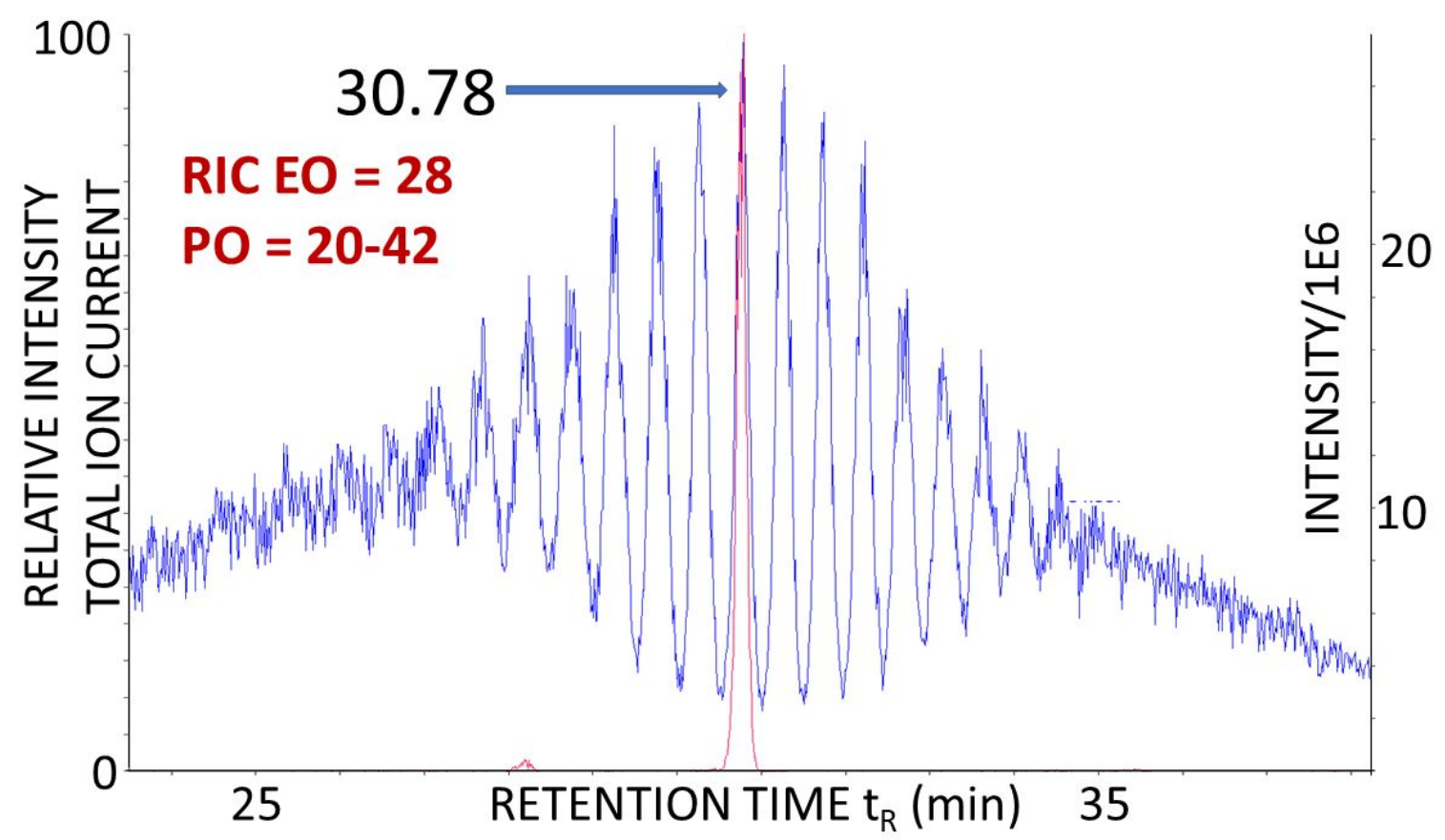

SI Fig 1 - SFC-(+) ion ESI FTMS analysis of the block oligomer PO-EO-PO, total ion current trace in blue from 10-28 minutes. Automatically generated reconstructed ion chromatogram (RICred) is shown as a result of a field of glyphs from the early-eluting impurity group $\mathrm{DBE}=1$ being selected on the interactive mass map. Compound identities were highlighted concomitantly on informational tables allowing ready assignment of the elution pattern. The series of "triplet" peaks are due to increasing ethoxylate (EO \# at SFC peak top) with a distribution of $\mathrm{PO}=2,3,4$ (decreasing peak intensity) in each EO group. 
Supplemental Information. Investigator Primary Processing Functions.

1. Mass Spectral Data Import and Raw Data Processing

Investigator directly imports mass spectral data from all major instrument manufacturers. The data formats include:

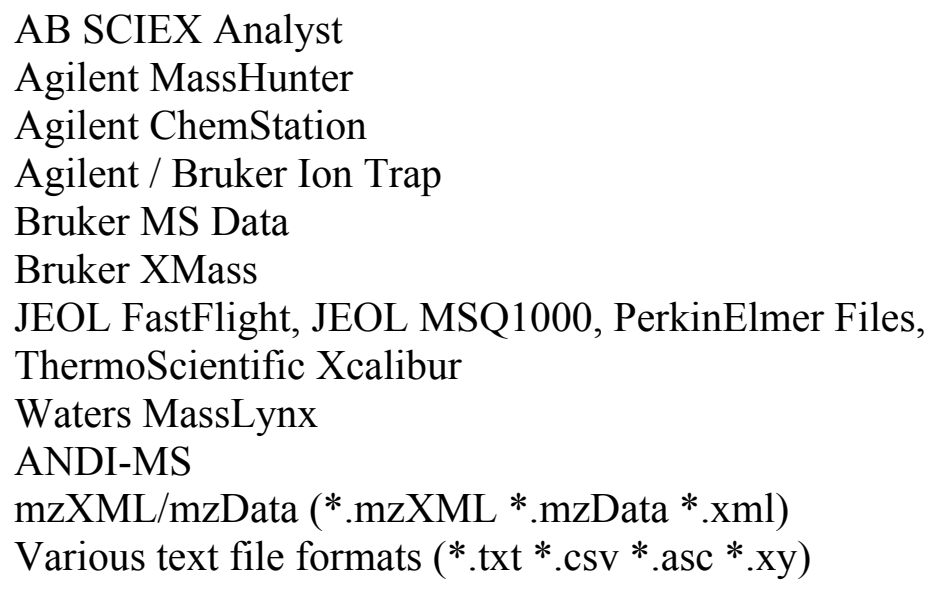

This section displays chromatographic traces and mass spectral data, facilitating spectra coaddition, background subtraction, and chromatographic trace displays of base peak and reconstructed ion chromatograms.

\section{Monoisotopic Detection}

Mass spectra are probed in detail to find all sets of isotope clusters and derived monoisotopic ions over the charge range specified by the user. Charge-deconvoluted neutral-mass spectra are produced.

\section{Mass Differences}

The mass differences between all pairs of monoisotopic masses up to a maximum mass difference specified by the user (typically $200 \mathrm{Da}$ ) are computed and are assigned neutral mass difference formulas and frequency of observation as the unique "mass differences spectrum". The plot interactively generates mass maps normalized to any repeating value found. Mass differences within isotope cluster ions are also determined, and can reveal the presence of certain $[\mathrm{A}, \mathrm{A}+2]$ element isotope pairs, e.g. $\left[{ }^{32} \mathrm{~S}\right],\left[{ }^{34} \mathrm{~S}\right]$ to "lock" formula requirements.

\section{Elemental Composition Assignment -}

A set of parameters, adjusted by the user, controls de novo elemental composition analysis of all detected $\mathrm{m} / \mathrm{z}$ values and the associated neutral monoisotopic masses. The parameters include charge state range, ionization modes, ionization adducts and/or losses, element ranges, DBE range, mass error limits, and recalibration operation. 
5. Molecular Formulae - the detailed results of assigning compositions to $\mathrm{m} / \mathrm{z}$ values, including ionization entities, are reduced to a list of single, representative neutral molecular formulae (which sometimes repeat in a table, i.e., if they are associated with multiple retention time apexes). The results are displayed in user-configured tables and graphs (2D, 3D maps as well as 2D reconstructed ion chromatograms). For 2D and 3D maps of any kind, the variable for each of the $\mathrm{X}, \mathrm{Y}$, and $\mathrm{Z}$ axes is selectable by the user, from a set of more than twenty items including: expressions of mass (e.g., m/z, neutral molecular weight, Kendrick-like KM and KMD variables), raw element counts and specific element ratios (e.g.VanKrevelen map) and several derived parameters like normalized isotope shift (NIS) and normalized mass defect NMD from Zubarev Maps, ref. [19]. The tables and graphs are interactive, i.e., selection of particular glyphs in a map causes highlighting of associated table information and generation of reconstructed ion chromatograms as in Figure 5. 